\title{
Delphi poll to assess consensus on issues influencing long-term adherence to treatments in cystic fibrosis among Italian health care professionals
}

This article was published in the following Dove Press journal:

Patient Preference and Adherence

\section{Carla Colombo' \\ Paola Catastini ${ }^{2}$ \\ Anna Brivio ${ }^{1,3}$ \\ Benedetto Acone ${ }^{4}$ \\ Patricia Dang 5 \\ Serena Quattrucci ${ }^{6}$ \\ 'Cystic Fibrosis Center, Fondazione IRCCS Ca' Granda, Ospedale \\ Maggiore Policlinico, Department of Pathophysiology and Transplantation, Università degli Studi di Milano, Milan, Italy; ${ }^{2}$ Cystic Fibrosis Regional Center, Anna Meyer Children's Hospital, Florence, Italy; ${ }^{3}$ UOC DPS Department for Health Professional, Fondazione IRCCS Ca' Granda, Ospedale Maggiore Policlinico, Milan, Italy; ${ }^{4}$ Cartesio Solutions S.r.I., Venice, Italy; ${ }^{5}$ Vertex Pharmaceuticals S.r.l., Rome, Italy; ${ }^{6}$ Cystic Fibrosis Lazio Regional Center, Pediatrics Department, "Sapienza" University, Rome, Italy}

Purpose: The aim of this study was to determine the level of consensus among Italian health care professionals (HCPs) regarding factors that influence adherence to cystic fibrosis (CF) treatments.

Methods: A Delphi questionnaire with 94 statements of potential factors influencing adherence was developed based on a literature review and in consultation with a board of experts $(n=4)$. This was distributed to a multidisciplinary expert panel of HCPs $(n=110)$ from Italian CF centers. A Likert scale was used to indicate the level of agreement $(1=$ no agreement to $9=$ maximum agreement) with each statement. Three rounds were distributed to establish a consensus ( $\geq 80 \%$ of participant ratings within one 3-point region) and, at the third round, assign a ranking to each statement with a high level of agreement (consensus in the 7-9 range) only.

Results: Of $110 \mathrm{HCPs}$ (from 31 Italian CF centers who were surveyed), responses were obtained from $85(77 \%)$ in the first, $78(71 \%)$ in the second, and $72(65 \%)$ in the third round. The highest degree of agreement (95.8\%) was reached with the statement that the HCP needs to build a relationship with the patient to influence adherence. A high level of agreement was not reached for statements that morbidity and mortality are influenced by the level of adherence to therapy, and no consensus was reached on the statement that age of the patient influences adherence to treatment.

Conclusion: We found that Italian HCPs endorsed a strong relationship with the patient as being a key driver in improving adherence. There were several areas, such as the influence of adherence on morbidity and mortality, where the consensus of Italian HCPs differed from the published literature. These areas require investigation to determine why these discrepancies exist.

Keywords: cystic fibrosis, long-term adherence, Delphi technique

\section{Introduction}

Cystic fibrosis (CF) is a lifelong, complex multisystem disease with significant challenges in treatment management. Treatments can be burdensome, time-consuming, and costly; $;^{1,2}$ the daily regimen can require ingestion of as many as 40-50 pills, inhalation treatments lasting up to 2 hours, and 2-3 airway clearance sessions of 30 minutes each. ${ }^{3}$ Equipment maintenance and preparation of medications, in addition to administrative barriers to maintaining access to medications, add to the time burden. ${ }^{4}$ As reported in the World Health Organization (WHO) document, ${ }^{5}$ poor adherence to long-term therapies severely compromises the effectiveness of treatment, making adherence a critical issue in the management of patients with chronic diseases.
Correspondence: Carla Colombo Cystic Fibrosis Center, Fondazione IRCCS Ca' Granda, Ospedale Maggiore Policlinico, Department of Pathophysiology and Transplantation, Università degli Studi di Milano, Via Della Commenda 9, 20122 Milan, Italy

Tel +390255032456

Fax +390255032814

Email carla.colombo@unimi.it 
Poor adherence is considered the single greatest cause of treatment failure, ${ }^{6}$ and results in increased morbidity and mortality, a reduction in quality of life, and increased health care use and costs. ${ }^{7,8}$ Adherence rates for $\mathrm{CF}$ treatments are generally below $50 \% ;{ }^{9,10}$ however, objective assessment tools are rarely used, with the vast majority of CF centers relying on clinical impression. ${ }^{11}$ In both children and adults with $\mathrm{CF}$, adherence decreases when the complexity of the regimen increases. ${ }^{12}$ Rates of adherence are higher with oral medications, lower with nebulized treatment and pancreatic enzymes, and lowest with vitamin treatment, dietary changes, exercise, and physiotherapy. ${ }^{13-18}$ In children whose parents strongly believe the treatment is necessary, better adherence is more likely. ${ }^{19}$

With improved patient survival, long-term management of CF has become an important focus, but treatment demands become repetitive and burdensome over the course of the disease, making long-term adherence challenging. ${ }^{13}$ Patients often carry out a personal cost-benefit analysis, assessing costs against the perceived necessity for, and their concerns about, their treatment regimen. ${ }^{19}$ In addition, patients with $\mathrm{CF}$ are faced with new challenges as they age, such as the transfer of responsibility for their medical treatment (from parents/caregivers to themselves) during the transition from adolescence to adulthood. ${ }^{20,21}$

In the recent update to the European Cystic Fibrosis Standards of Care: Best Practice Guidelines in the treatment of $\mathrm{CF}$, the core components to addressing adherence were determined to be: team ethos with respect to patient care, collaboration with patients, identification of the barriers to adherence, and active support of patients' efforts. ${ }^{22}$ Adherence to treatment regimens can be influenced by many emotional factors, including dependency, feeling different, embarrassment at taking drugs in public places, effect on personal freedom, and significant influence on lifestyle. Usually, the focus is on patient-related factors, while the provider- and health system-related determinants of nonadherence, which can have a major effect, are neglected. ${ }^{23}$

The aim of this multicenter study was to investigate the level of consensus among Italian health care professionals (HCPs) on issues identified in the literature as influencing adherence to treatments in patients with CF. The study sought to identify areas of consensus and disagreement with the literature. The goal was to identify educational needs among Italian HCPs in order to develop a program of instruments, actions, and operational modalities (applicable in clinical practice) to support and enhance patients' long-term adherence to treatment.

\section{Methods}

The Delphi process, developed in the $1950 \mathrm{~s},{ }^{24}$ is a communication process widely used to establish consensus among experts when there is insufficient evidence to determine an objective answer. The process has been widely applied to health-related research, ${ }^{25,26}$ and involves a panel of experts anonymously completing a series of structured questionnaires, with the responses provided to the participants between rounds and amended in subsequent rounds, until a consensus is reached. The structure of the process is designed to allow group consensus without direct confrontation and to allow participants to gather opinions and react in subsequent rounds. Review and approval of this study by an institutional review board or ethics committee were not required as no patient data were obtained. By completing and returning the questionnaire, each participant consented to being involved in the study.

We first established a multidisciplinary expert board (two physicians, one psychologist, and one physiotherapist) and then implemented a bibliographic search of articles in PubMed, published in English language journals after January 1, 1995, using the keywords "compliance" OR/AND "adherence" AND "cystic fibrosis" OR/AND "cystic fibrosis therapy" AND "motivational interviewing" OR/AND "physician-patient relations". Thirty-one papers were identified (one randomized controlled trial, 17 observational studies, six reviews, five systematic reviews, one opinion, and one state-of-the-art review) and sent to the members of the expert board.

Our review of this collection of manuscripts identified 165 statements related to treatment adherence among patients with $\mathrm{CF}$, which were used to create a questionnaire (in Italian). No pilot testing was conducted; however, each assumption was evaluated three times, first independently by each member of the expert board via e-mail, followed by two collective teleconferences, with a final meeting to refine and validate each assumption. At the end of the selection process, duplications and redundancies were eliminated, and 94 statements were considered for the Delphi questionnaire and divided into three areas: "General Aspects" (six categories), "Roles and Relational Aspects" (three categories), and "Management Aspects" (four categories). A Likert scale was used by the respondents to evaluate the level of agreement with each of the statements in the questionnaire $(1=$ no agreement to $9=$ maximum agreement). All activities were coordinated by a facilitator.

All Italian specialized centers dedicated to care and management of patients with CF were contacted and asked for volunteer participation from different HCPs who met 
the following criteria, as identified by the multidisciplinary expert board:

- At least 2-years' experience in the field (for physicians)

- In the last 2 years, at least $50 \%$ of their weekly work time dedicated to patients with CF (for non-physicians)

The questionnaire was sent by e-mail with a maximum of three reminders; the answers arrived via e-mail, fax, or postal mail.

Definitions for consensus and no consensus were decided a priori based on prior literature. ${ }^{27,28}$ Consensus was defined as $\geq 80 \%$ of participant ratings within one 3 -point region $(1-3=$ low level of agreement; $4-6=$ borderline; $7-9=$ high level of agreement). Disagreement was defined as $\geq 90 \%$ of participant ratings within one of two wide ranges (1-6 or 4-9). Results outside the ranges for consensus and disagreement were defined as no consensus. The collected assessments were evaluated for internal consistency and aggregated to obtain a composite judgment.

The HCP panel was consulted three times in total. In the first round, the questionnaire was distributed and the level of agreement among HCPs in relation to each statement was determined. In the second round, statements for which there was disagreement during the first round were shared with the HCP panel, who were allowed to alter their responses from the first round. A third round of consultation was conducted to apply rankings to each of the statements that had a high level of agreement following rounds one and two. Rankings were assigned within each of the three areas with one statement defined as the highest rank.

The flow chart of the analysis is presented in Figure 1. Calculations for the analysis were performed using Microsoft Excel 2007 software package (Microsoft Corporation, Redmond, WA, USA).

\section{Results}

The analysis was conducted in Italy from January 2015 to June 2015. All 32 CF centers in Italy were contacted and a total of 110 HCPs from 31 centers participated in the study. The distribution of HCP categories responding in the first round $(n=85)$ is presented in Figure 2; the inclusion criteria for volunteers resulted in the participation of a multidisciplinary expert panel of HCPs. Although participation in the study was purely voluntary, the response rate remained high throughout. Responses were obtained from 85 HCPs (77\%) in the first round and $78(71 \%)$ and $72(65 \%)$ HCPs in the second and third rounds, respectively.

Participation was lower among physicians (64\%) and higher among nurses (93\%), physiotherapists (95\%), and

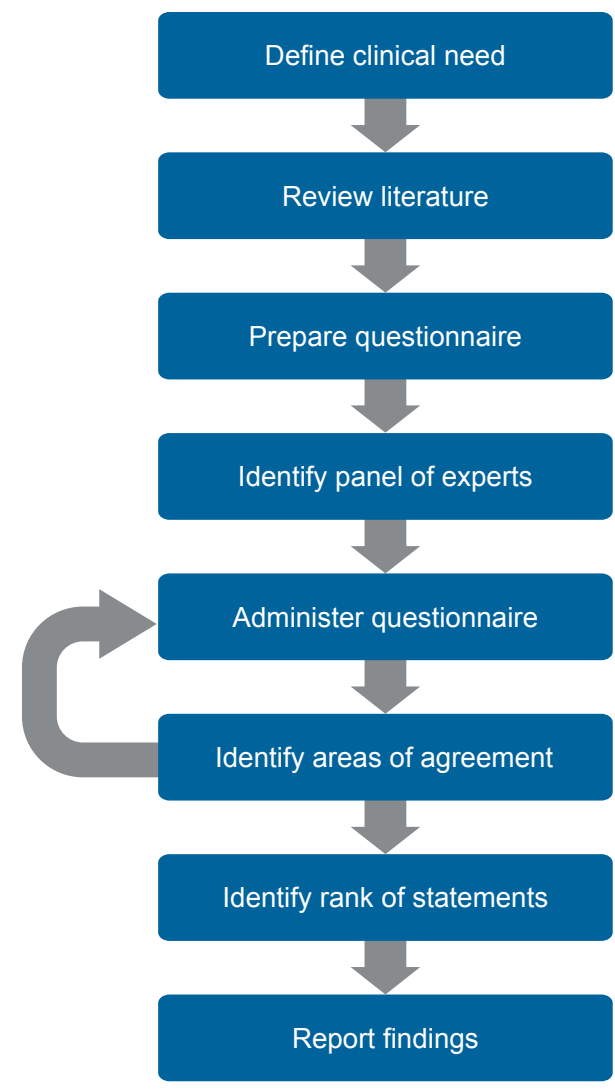

Figure I Process flow chart.

Note: Calculations for the analysis were performed using Microsoft Office 2007 software package (Microsoft Corporation, Redmond, WA, USA).

psychologists (100\%). The distribution of respondents in the second and third round did not vary greatly when compared with the first round, with physicians' participation at 59\% in the second round and 55\% in the third round, nurses' participation at $86 \%$ in both rounds, physiotherapists' participation

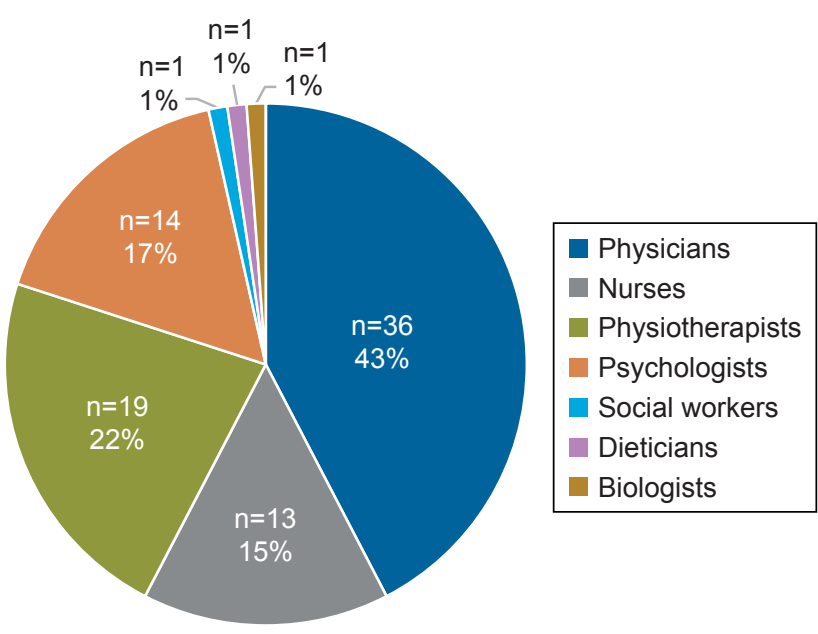

Figure 2 Distribution of HCP categories among responders to the first round of the Delphi analysis (total number of responders $=85$ ).

Abbreviation: HCP, health care provider. 
at $80 \%$ and $75 \%$, and psychologists' participation at $100 \%$ and $71 \%$, respectively.

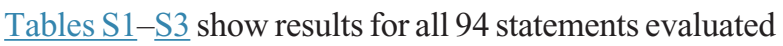
in the first and second round, and the rankings assigned (for statements with high-level agreement).

A high level of agreement was obtained from the first round on 37 statements ( $\geq 80 \%$ of responses in regions $7-9$ ) and a low level of agreement ( $\geq 80 \%$ of responses in regions 1-3) only on one statement. After the second round, level of agreement was similar to the first round; therefore, a third round was conducted only to assign a rank to the 40 statements with a high level of agreement (seven in "General Aspects", 12 in "Roles and Relational Aspects", and 21 in "Management Aspects"), representing 45\% of all statements.

Figure 3 presents the ranked scores for statements in the "General Aspects" area. The highest level of agreement (first-level ranking) was the statement "Adherence means agreeing to one's own treatment plan and committing to follow it" (83.1\%). Of interest, the treating team's communication skills were deemed more important (in terms of influencing adherence to treatment in patients with $\mathrm{CF}$ ) than a physician's ability to communicate.

Statements assigned a rank in the "Roles and Relational Aspects" area are presented in Figure 4. A very high level of agreement (95.8\%) was reached on the statement affirming that building a relationship with the patient is necessary to influence treatment adherence, which was assigned a firstlevel ranking. All the statements in the "Roles and Relational Aspects" area confirmed the following: the perception of the important role of individual HCPs as well as treating the HCP team as a whole; the accuracy of information conveyed to the patient; the patient's comprehension of that information and the patient guiding change; the ability to listen and to discuss; the need to share the interventions dedicated to supporting adherence and for interventions to be consistent; and the need to define treatment objectives step by step.

Statements assigned a rank in the "Management Aspects" area are presented in Figure 5. Of note is the high level of agreement among all HCPs on almost all statements in the "Management Aspects" area (21 out of 29 statements). The quality of communication and the need to have a structured

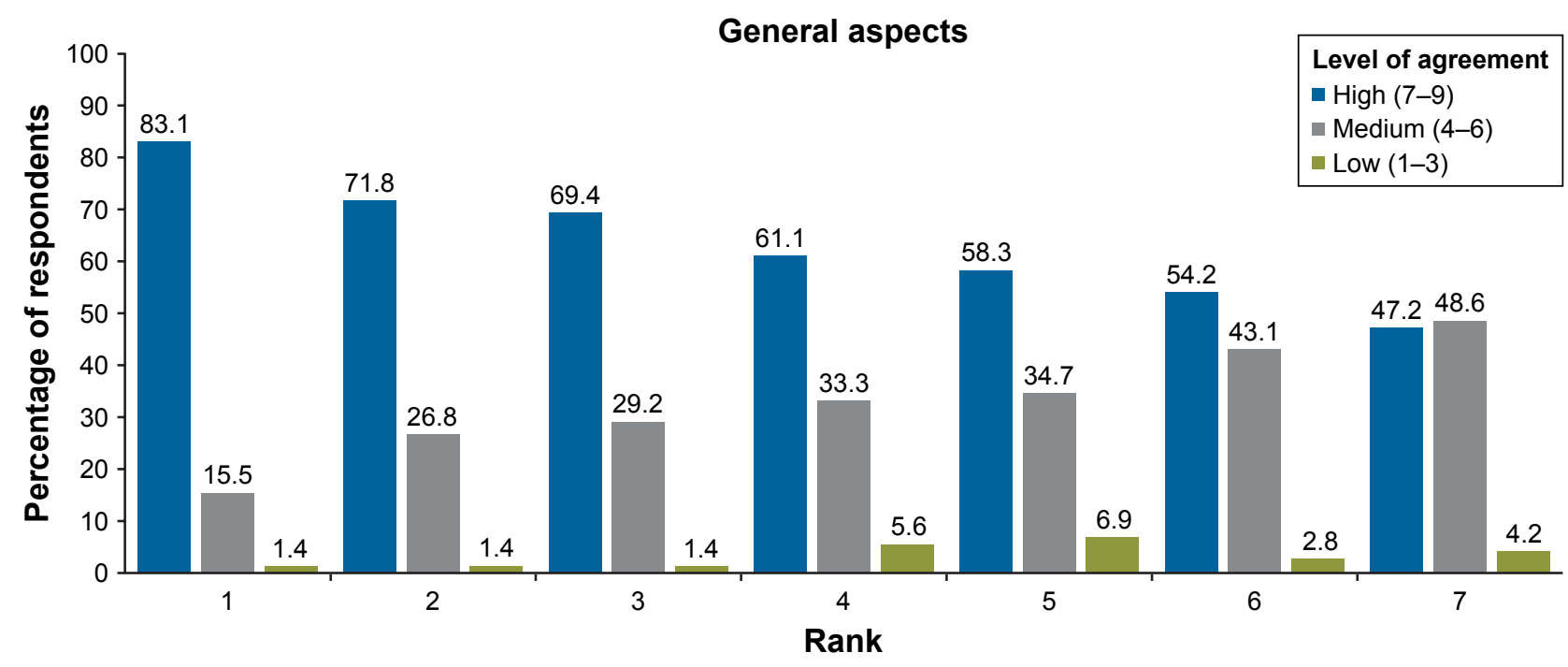

\begin{tabular}{ll}
\hline Rank & Statement \\
\hline 1 & $\begin{array}{l}\text { Adherence means agreeing to one's own treatment plan and committing to follow it } \\
2\end{array}$ \\
$\begin{array}{l}\text { Adherence is influenced by the difficulty in combining the patient's lifestyle and obligations } \\
\text { imposed by therapies }\end{array}$ \\
3 & $\begin{array}{l}\text { Adherence is an individual behavior comprising the degree of concordance with the medical advice } \\
\text { received, and the persistence of the behavior itself (ie, continued treatment for the recommended duration) }\end{array}$ \\
4 & Adherence is influenced by the high burden of treatment \\
5 & Adherence is conditioned by psycho-emotional conditions \\
6 & Adherence is influenced by the treating team's communication skills \\
7 & Adherence is influenced by the physician's ability to communicate \\
\hline
\end{tabular}

Figure 3 Ranking of statements with a high level of agreement in the "General Aspects" area. Note: Results from all 38 statements are provided in Table SI. 


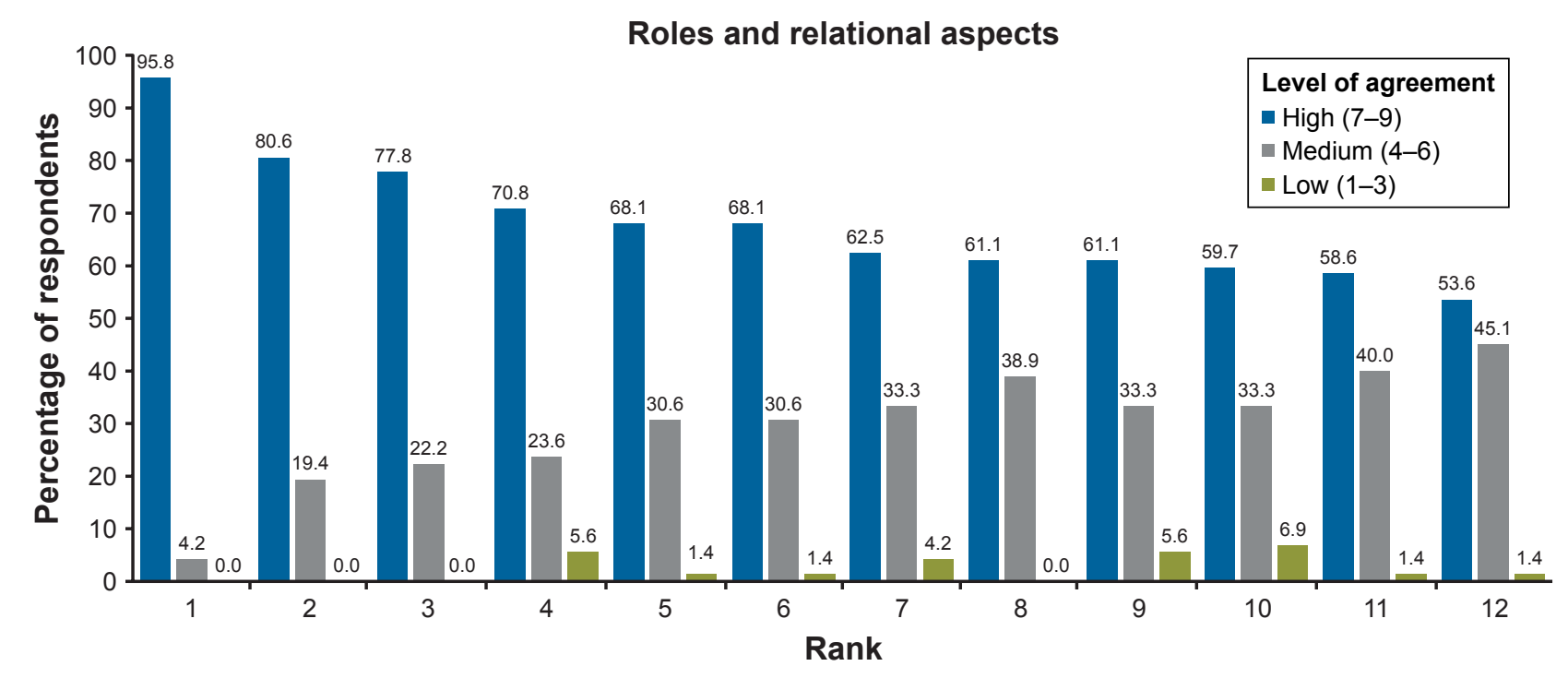

\begin{tabular}{ll}
\hline Rank & Statement \\
\hline 1 & To influence adherence, it is necessary to build a relationship with the patient \\
2 & Adherence is influenced by the understanding and sharing of interventions dedicated to its support \\
3 & To improve adherence, it is helpful to have an open and gradual approach to dialogue \\
4 & Listening to the patient attitude (what does he do? why?) increases adherence \\
5 & Adherence is influenced by the accuracy of information received by the patient from the care team \\
6 & To influence adherence, it is necessary to understand the reasons behind the obstacles experienced by patients \\
7 & To improve adherence, a structured motivational approach is useful \\
8 & Adherence is influenced by the consistency of interventions dedicated to its support \\
9 & The ability to influence adherence increases with understanding (what does he know? what does he want?) \\
10 & The patient should be put in a situation where he can guide his own change \\
11 & Adherence is influenced by HCP's ability to explain multiple aspects of the treatment \\
12 & Adherence is influenced by the accuracy of information received by the patient from the physician \\
\hline
\end{tabular}

Figure 4 Ranking of statements with a high level of agreement in the "Roles and Relational Aspects" area.

Note: Results from all 27 statements are provided in Table S2.

Abbreviation: $\mathrm{HCP}$, health care provider.

and consistent approach through a personalized, collaborative, open dialogue with the patient were unanimously evaluated as important.

All statements, including those that did not reach a consensus, are presented in Tables $\mathrm{S} 1-\mathrm{S} 3$. In addition, differences in opinion between HCPs (as identified in the first round) are presented in Tables S4-S6. The level of agreement for statements regarding morbidity and mortality being influenced by adherence to therapy was lower than for other items (such as those describing both internal and external factors influencing adherence); different levels of agreement were found between the different professionals in the treating team. Physicians tended to agree more than other HCPs that morbidity is influenced by adherence. Consensus was not reached for the statements that the age of the patient influences adherence to treatment, or that adherence is a problem for the adolescent patient.
Among the "Management Aspects", 14\% of the HCPs surveyed reached a low agreement (responses in regions 1-3) on the issue of "understanding without judging the patient".

\section{Discussion}

Various barriers to adherence to $\mathrm{CF}$ treatments have been described, including lack of time, forgetfulness, unwillingness to take medication in public, high level of polypharmacy, poor patient-HCP communication, lack of disease- and treatment-related knowledge, and the patient's or caregiver's beliefs. ${ }^{29}$ The Italian HCPs surveyed in our study reached a high level of agreement with almost half of the factors that influence adherence identified in the literature. These findings are important in order to provide suggestions about new interventional studies, educational materials needed for HCPs, and operational modalities applicable in clinical 


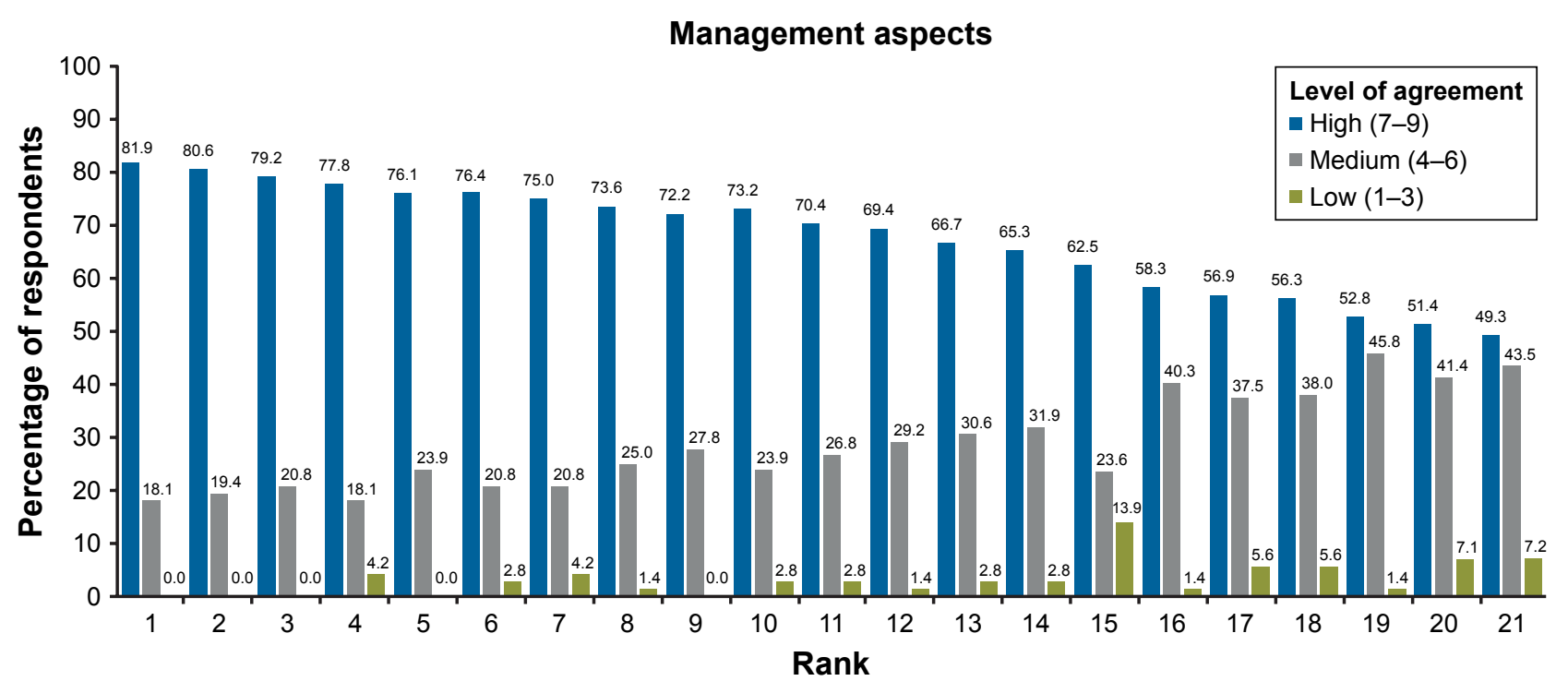

\begin{tabular}{ll}
\hline Rank & Statement \\
\hline 1 & Priority is to favor the patient's (and caregiver) involvement \\
2 & Quality of communication means listening and understanding the expectations and beliefs of the patient \\
3 & Priority is to pay attention to the patient and his needs \\
4 & Quality of communication means being able to find the motivation and the patient involvement \\
5 & The quality of communication between the team, the patient, and the family improves adherence because it makes \\
& the patient (and the caregiver) feel part of the team \\
6 & Creating time to share positive information and appreciating the results obtained influence adherence \\
7 & Patients have a higher probability of adherence when they feel emotionally supported by the physician and by the HCP team \\
8 & A communication-oriented approach to problem solving improves adherence \\
9 & Quality of communication means being able to use the progress to consolidate the adherence \\
10 & Quality of communication means negotiating gradual and achievable goals (step by step) \\
11 & Quality of communication means helping to make informed choices \\
12 & The team should strive for sincerity and openness from patients and families in order to receive quality information \\
13 & to improve adherence \\
14 & The patient's expectations play an important role in the management of adherence \\
15 & Quality of communication means to understand without criticizing (make judgments) \\
16 & Quality of communication means being able to measure and reward progress \\
17 & Quality of communication means trying to speak the same language \\
18 & The treating team should adopt a structured method of communication (questions, listening, paraphrasing, etc.) \\
19 & Building and consolidating the strengths within the family influences adherence \\
20 & Patients have a higher probability of adherence when they feel emotionally supported by the physician \\
21 & Making perceptible the fact that the HCP understands how the patient is living with his condition influences adherence
\end{tabular}

Figure 5 Ranking of statements with a high level of agreement in the "Management Aspects" area.

Note: Results from all 29 statements are provided in Table S3.

Abbreviation: HCP, health care provider.

practice to support and enhance patient's long-term adherence to treatment.

A strong relationship between the patient and the CFtreating team was endorsed as a key driver of improved adherence in previous studies ${ }^{30,31}$ and by the Italian HCPs surveyed. Establishing effective communication and interaction between patients, their families, and caregivers is one of the most important, and potentially one of the simplest, approaches to increase adherence in CF. Even considering the distinct functions of the different HCPs in treating CF, focusing on the patient rather than the disease can reduce differences in the perceptions of what each HCP can do to encourage and support the patient's adherence to treatments in the long term. In a large meta-analysis of studies conducted between 1949 and 2008, the probability of adherence was 2.1 times greater for patients treated by a physician who was classified as a good communicator. ${ }^{32}$ A collaborative approach centered on patient care was also found to be 
important in motivating patients. ${ }^{31,33}$ New approaches to training and training activities to increase competencies in the use of novel patient-centered tools, such as the motivational interview, ${ }^{34}$ are important, and needed, but should be investigated through interventional studies incorporating them into daily standard clinical activities. Although a high level of agreement was reached between HCPs after the second round, representing $45 \%$ of all statements, agreement was not obtained for the remaining statements (eg, "The mortality/ morbidity of patients with cystic fibrosis is influenced by the level of adherence to the therapeutic prescriptions received" and "Adherence is influenced by the age of the patient"). It is useful to determine on which statements HCPs did not reach an agreement, as this can stimulate interest and inform future discussions and the development of focused training plans. However, in some instances, the low level of agreement among HCPs could be a result of differences in the interpretation of questions or slight semantic differences. There were also some notable discrepancies between the level of agreement among the Italian HCPs surveyed and the published literature. The influence of adherence on morbidity and mortality has been well described, ${ }^{8}$ as has the fact that younger patients tend to have higher rates of treatment adherence than adolescents and adults. ${ }^{35}$ However, in our study of Italian HCPs, a high level of agreement was not reached on these statements. These discrepancies could be explained by the differences in roles within the treating team; physicians oversee the management of patients with $\mathrm{CF}$ and seem more conscious of morbidity outcomes and the overall consequences of low adherence, while other HCPs, such as physiotherapists and psychologists, are focused on specific aspects of the management of CF patients. Furthermore, the views of Italian HCPs could be influenced by local factors, such as the Italian education system and cultural beliefs and/or values, which could account for the discrepancies observed. Additional research will be necessary to determine why these discrepancies exist between the opinions of HCPs and the published literature and to develop educational programs and training materials to ensure that HCPs are aware of the influence of these factors on adherence to treatment.

\section{Limitations}

Our descriptive, non-interventional study has several limitations. First, the multidisciplinary expert board consisted of only four HCPs; however, one of these HCPs is head of the Società Italiana Di Fibrosi Cistica (SIFC, a national scientific society) working group on adherence, of which the remaining three HCPs are all members. This was agreed to be sufficient for the purposes of this study. Second, the HCPs surveyed represent a self-selected population that is engaged enough to commit to three rounds of surveys and may not be representative of other HCPs. Although HCPs were engaged, participation rates decreased slightly between rounds, which may be due to reasons such as attrition, reduced personal motivation, or the amount of time required to complete the requested rounds of consultation. While the participation rate was lower among physicians than with psychologists, the physician participation rate was still relatively high, with the difference observed between professions possibly due to greater motivation among psychologists to complete questionnaires on this topic. We also only included HCPs from Italian CF centers; therefore, the results cannot necessarily be applied to other European or North American CF centers. Adherence was not explicitly defined, although several statements addressed how the HCPs defined adherence (eg, "agreeing to one's own treatment plan and committing to follow it"; "an individual behavior comprising the degree of concordance with the medical advice received"). Furthermore, the Delphi poll measured HCPs' beliefs about what affected long-term medication adherence. However, medication adherence is a patient behavior, and not a HCPrelated behavior. Therefore, the actual driving force behind medication adherence may be different to that considered by HCPs in determining why patients continue to take their medications, or not. An additional limitation is that there is no universally agreed definition of consensus, with several factors, such as the number of respondents, aim of the research, and resources, influencing the cutoff. ${ }^{26}$ Even with these limitations, the Delphi technique has been established as a valuable means for structuring group discussion among experts and raising issues for debate.

\section{Conclusion}

We have identified important areas of consensus and disagreement regarding factors that influence adherence to CF treatments among Italian HCPs. To Italian physicians, adherence generally means the patient agrees to and commits to following a specific treatment plan; a strong physicianpatient relationship is a key factor in influencing adherence. More standard measures of adherence (medication possession ratio or proportion of days covered) rely solely on a patient's medication refill history and do not account for additional factors that might influence adherence (eg, the patient taking the medication in the correct way). These results are a first step in developing training tools and educational materials to work with HCPs to improve the overall adherence to 
treatment, which can ultimately lead to improved long-term outcomes.

\section{Acknowledgments}

Medical writing and editorial support were provided by Stephanie Vadasz, PhD, and Dena McWain. SV and DM are employees of Ashfield Healthcare Communications, which received funding from Vertex Pharmaceuticals Incorporated. The Delphi survey described in this manuscript was carried out by Cartesio Solutions S.r.1. and funded by Vertex Pharmaceuticals S.r.l.

\section{Author contributions}

All authors made substantial contributions to 1) the conception and design of the study, or acquisition of data, or analysis and interpretation of data, 2) drafting the article or revising it critically for important intellectual content, 3) final approval of the version to be submitted, and 4) agree to be accountable for all aspects of the work.

\section{Disclosure}

$\mathrm{CC}, \mathrm{PC}, \mathrm{AB}, \mathrm{BA}$, and SQ received fees from Vertex Pharmaceuticals S.r.l. for preparation of the Delphi questionnaire. $\mathrm{CC}$ received fees to serve on advisory boards for Vertex Pharmaceuticals, Mylan, Novartis, and Teva. PC, AB, and SQ received fees to serve on advisory board(s) for Vertex Pharmaceuticals S.r.1. AB received fees as a speaker for Teva Italia S.r.1. BA received fees as a speaker for Vertex Pharmaceuticals S.r.1. PD is an employee of Vertex Pharmaceuticals S.r.l., and owns stock/stock options in that company. The authors report no other conflicts of interest in this work.

\section{References}

1. Ziaian T, Sawyer MG, Reynolds KE, et al. Treatment burden and healthrelated quality of life of children with diabetes, cystic fibrosis and asthma. J Paediatr Child Health. 2006;42(10):596-600.

2. Sawicki GS, Sellers DE, Robinson WM. High treatment burden in adults with cystic fibrosis: challenges to disease self-management. J Cyst Fibros. 2009;8(2):91-96.

3. Marciel KK, Saiman L, Quittell LM, Dawkins K, Quittner AL. Cell phone intervention to improve adherence: cystic fibrosis care team, patient, and parent perspectives. Pediatr Pulmonol. 2010;45(2):157-164.

4. Geller DE, Madge S. Technological and behavioral strategies to reduce treatment burden and improve adherence to inhaled antibiotics in cystic fibrosis. Respir Med. 2011;105(Suppl 2):S24-S31.

5. Sabate E. Adherence to Long-Term Therapies: Evidence for Action. Geneva, Switzerland: World Health Organization; 2003. Available from: http://www.who.int/chp/knowledge/publications/adherence_report/en/. Accessed July 20, 2018.

6. Quittner AL, Modi AC, Lemanek KL, Ievers-Landis CE, Rapoff MA. Evidence-based assessment of adherence to medical treatments in pediatric psychology. J Pediatr Psychol. 2008;33(9):916-936; discussion 937-938.

7. O'Donohoe R, Fullen BM. Adherence of subjects with cystic fibrosis to their home program: a systematic review. Respir Care. 2014;59(11): 1731-1746.
8. Eakin MN, Riekert KA. The impact of medication adherence on lung health outcomes in cystic fibrosis. Curr Opin Pulm Med. 2013;19(6): 687-691.

9. Modi AC, Lim CS, Yu N, Geller D, Wagner MH, Quittner AL. A multi-method assessment of treatment adherence for children with cystic fibrosis. J Cyst Fibros. 2006;5(3):177-185.

10. Sawicki GS, Tiddens H. Managing treatment complexity in cystic fibrosis: challenges and opportunities. Pediatr Pulmonol. 2012;47(6): 523-533.

11. Riekert KA, Eakin MN, Bilderback A, Ridge AK, Marshall BC. Opportunities for cystic fibrosis care teams to support treatment adherence. J Cyst Fibros. 2015;14(1):142-148.

12. Sawicki GS, Ren CL, Konstan MW, Millar SJ, Pasta DJ, Quittner AL; Investigators and Coordinators of the Epidemiologic Study of Cystic Fibrosis. Treatment complexity in cystic fibrosis: trends over time and associations with site-specific outcomes. J Cyst Fibros. 2013;12(5): 461-467.

13. White D, Stiller K, Haensel N. Adherence of adult cystic fibrosis patients with airway clearance and exercise regimens. J Cyst Fibros. 2007;6(3): $163-170$.

14. Myers LB, Horn SA. Adherence to chest physiotherapy in adults with cystic fibrosis. J Health Psychol. 2006;11(6):915-926.

15. Nasr SZ, Chou W, Villa KF, Chang E, Broder MS. Adherence to dornase alfa treatment among commercially insured patients with cystic fibrosis. J Med Econ. 2013;16(6):801-808.

16. Zindani GN, Streetman DD, Streetman DS, Nasr SZ. Adherence to treatment in children and adolescent patients with cystic fibrosis. $J$ Adolesc Health. 2006;38(1):13-17.

17. Anthony H, Paxton S, Bines J, Phelan P. Psychosocial predictors of adherence to nutritional recommendations and growth outcomes in children with cystic fibrosis. J Psychosom Res. 1999;47(6):623-634.

18. Duff AJ, Latchford GJ. Inhaled medication and inhalation devices for lung disease in patients with cystic fibrosis: poor adherence and the need to address it. J Cyst Fibros. 2010;9(6):455-456.

19. Goodfellow NA, Hawwa AF, Reid AJ, Horne R, Shields MD, McElnay JC. Adherence to treatment in children and adolescents with cystic fibrosis: a cross-sectional, multi-method study investigating the influence of beliefs about treatment and parental depressive symptoms. BMC Pulm Med. 2015;15:43.

20. Bregnballe V, Schiøtz PO, Lomborg K. Parenting adolescents with cystic fibrosis: the adolescents' and young adults' perspectives. Patient Prefer Adherence. 2011;5:563-570.

21. Hobbs SA, Schweitzer JB, Cohen LL, Hayes AL, Schoell C, Crain BK. Maternal attributions related to compliance with cystic fibrosis treatment. J Clin Psychol Med Settings. 2003;10(4):273-277.

22. Smyth AR, Bell SC, Bojcin S, et al; European Cystic Fibrosis Society. European Cystic Fibrosis Society Standards of Care: Best Practice guidelines. J Cyst Fibros. 2014;13(Suppl 1):S23-S42.

23. Osterberg L, Blaschke T. Adherence to medication. $N$ Engl J Med. 2005;353(5):487-497.

24. Dalkey NC, Helmer-Hirschberg O. An Experimental Application of the Delphi Method to the Use of Experts. Santa Monica, CA: Rand Corp; 1962.

25. de Meyrick J. The Delphi method and health research. Health Educ. 2003;103(1):7-16.

26. Hasson F, Keeney S, McKenna H. Research guidelines for the Delphi survey technique. J Adv Nurs. 2000;32(4):1008-1015.

27. Lapadula G, Marchesoni A, Salaffi F, et al. Evidence-based algorithm for diagnosis and assessment in psoriatic arthritis: results by Italian DElphi in psoriatic Arthritis (IDEA). Reumatismo. 2016;68(3):126-136.

28. Linder D, Altomare G, Amato S, et al. PSOCUBE, a multidimensional assessment of psoriasis patients as a both clinically/practically sustainable and evidence-based algorithm. JEur Acad Dermatol Venereol. 2015; 29(7):1310-1317.

29. Narayanan S, Mainz JG, Gala S, Tabori H, Grossoehme D. Adherence to therapies in cystic fibrosis: a targeted literature review. Expert Rev Respir Med. 2017;11(2):129-145. 
30. Lomas P. Enhancing adherence to inhaled therapies in cystic fibrosis. Ther Adv Respir Dis. 2014;8(2):39-47.

31. Sawicki GS, Heller KS, Demars N, Robinson WM. Motivating adherence among adolescents with cystic fibrosis: youth and parent perspectives. Pediatr Pulmonol. 2015;50(2):127-136.

32. Zolnierek KB, Dimatteo MR. Physician communication and patient adherence to treatment: a meta-analysis. Med Care. 2009;47(8): 826-834.

33. Dwamena F, Holmes-Rovner M, Gaulden CM, et al. Interventions for providers to promote a patient-centred approach in clinical consultations. Cochrane Database Syst Rev. 2012;12:CD003267.
34. Duff AJ, Latchford GJ. Motivational interviewing for adherence problems in cystic fibrosis; evaluation of training healthcare professionals. J Clin Med Res. 2013;5(6):475-480.

35. Vandenbranden SL, McMullen A, Schechter MS, et al; Investigators and Coordinators of the Epidemiologic Study of Cystic Fibrosis. Lung function decline from adolescence to young adulthood in cystic fibrosis. Pediatr Pulmonol. 2012;47(2):135-143.

\section{Publish your work in this journal}

Patient Preference and Adherence is an international, peer-reviewed, open access journal that focuses on the growing importance of patient preference and adherence throughout the therapeutic continuum. Patient satisfaction, acceptability, quality of life, compliance, persistence and their role in developing new therapeutic modalities and compounds to optimize clinical outcomes for existing disease states are major areas of interest for the journal. This journal has been accepted for indexing on PubMed Central. The manuscript management system is completely online and includes a very quick and fair peer-review system, which is all easy to use. Visit http://www. dovepress.com/testimonials.php to read real quotes from published authors.

\footnotetext{
Submit your manuscript here: http://www.dovepress.com/patient-preference-and-adherence-journal
} 